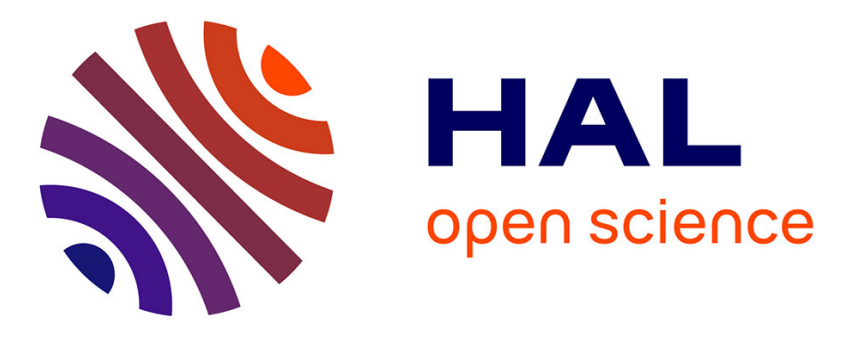

\title{
Characterization of whole plant leaf area properties using laser scanner point clouds
}

Gaëtan Louarn, Serge Carré, Frédéric Boudon, Annie Eprinchard, Didier Combes

\section{- To cite this version:}

Gaëtan Louarn, Serge Carré, Frédéric Boudon, Annie Eprinchard, Didier Combes. Characterization of whole plant leaf area properties using laser scanner point clouds. Fourth International Symposium on Plant Growth Modeling, Simulation, Visualization and Applications, 2012, Shanghai, China. hal00828841

\section{HAL Id: hal-00828841 https://hal.inria.fr/hal-00828841}

Submitted on 31 May 2013

HAL is a multi-disciplinary open access archive for the deposit and dissemination of scientific research documents, whether they are published or not. The documents may come from teaching and research institutions in France or abroad, or from public or private research centers.
L'archive ouverte pluridisciplinaire HAL, est destinée au dépôt et à la diffusion de documents scientifiques de niveau recherche, publiés ou non, émanant des établissements d'enseignement et de recherche français ou étrangers, des laboratoires publics ou privés. 


\title{
Characterization of whole plant leaf area properties using laser scanner point clouds
}

\author{
Louarn Gaëtan $^{1 *}$, Carré Serge ${ }^{1}$, Boudon Frédéric ${ }^{2}$, Eprinchard Annie ${ }^{1}$, Combes Didier ${ }^{1}$ \\ ${ }^{1}$ INRA UR4 P3F, BP6, F686600 Lusignan, France \\ ${ }^{2}$ CIRAD/INRIA, Virtual Plants INRIA Team, UMR AGAP, TA A-108/02, 34398 Montpellier \\ Cedex 5, France \\ * gaetan.louarn\{a\}lusignan.inra.fr
}

\begin{abstract}
Whole plant leaf area properties were extracted from contrasting laser scanner point clouds of herbaceous plants and compared to information obtained by $3 D$ magnetic digitization. Total leaf area and its spatial distribution were strongly related to the total number of scanned points and its spatial distribution. The calculations of normal directions allowed, to some extent, to infer the distribution of plant surfaces elevation angles. Together with the definition of an accurate plant boundary envelope, these data allowed the calculation of light interception as accurately as magnetic digitization by using simpler turbid medium plant representations.
\end{abstract}

\section{Introduction}

High-throughput screening of many genotypes and the phenotypic analysis of their response to environmental conditions has become a major challenge to unravel the genetic basis of complex traits in plants [1]. In this context, automating the interpretation and analysis of biological images is of particular importance. Current advances in 3D imaging are likely to provide performing tools in a near future to deal with these phenotyping issues [2, 3]. Laser scanner devices in particular can generate valuable data to infer the topology and geometry of plants [4, 5]. Much work however remains to be done to develop algorithm making full use of the information available in the 3D point clouds generated [5, 6].

At the same time, it has been recently pointed out that complex plant architectures can be summarized through a limited set whole-plant variables (i.e. plant boundary envelope, total leaf area, leaf angle distribution and a characterization of leaf area clumping) to infer key plant function such as light interception and carbon uptake [7, 8]. A partial use of the full geometric information might thus be enough for the phenotyping of these functions. The plant representation used in that case relies on a 'turbid medium' analogy (i.e. plant structure seen as a homogeneous medium of random small particles within the plant boundary envelope) to which a supplemental leaf dispersion parameter can be introduced to empirically deal with the non-random clumping of foliage. Relative vertical and horizontal leaf area distributions were shown to advantageously replace this leaf dispersion parameter in herbaceous plants [8].

The objectives of the present paper were i) to assess if whole plant features necessary to infer light interception through simple plant turbid medium (i.e. plant boundary envelope, total leaf area, leaf angle distribution and spatial distribution of leaf area) could be extracted from laser scanner point clouds and ii) to validate the accuracy of these data on light interception calculation. A series of herbaceous plants was scanned and then digitized with a magnetic digitization device to build 3D reference plants and allows comparison of laser scanner data with a reference mesh of each plant.

\section{Materials and Methods}

\subsection{Plant material studied}

An experiment was carried out outdoors between April and September 2009 at the INRA Lusignan station, France. Alfalfa (Medicago sativa L. cv Orca) was sown at 50 (LD) and 460 (HD) plants.m ${ }^{-2}$. Each plant was grown in an individual pot to facilitate extraction of individual plants from the canopy. Pots were ferti-irrigated three times a day with a complete nutrient solution. 


\subsection{Three-dimensional digitising}

For each density, 3D digitizations were performed at two stages of development (once during vegetative development D1, and once at the end of a summer regrowth period D2) on a group of neighbouring plants extracted from the centre of each canopy. They were measured indoors using an electromagnetic 3D digitizer (3Space Fastrak, Polhemus Inc., Colchester, VT, USA). Detailed record of the multi-scale topology of each plant and of the spatial coordinates and geometry of all plant organs were performed as described in $[9,10]$. The reconstruction process of triangulated virtual plants was achieved using the PlantGL toolkit [11] in the Openalea platform [12] (Fig. 1).

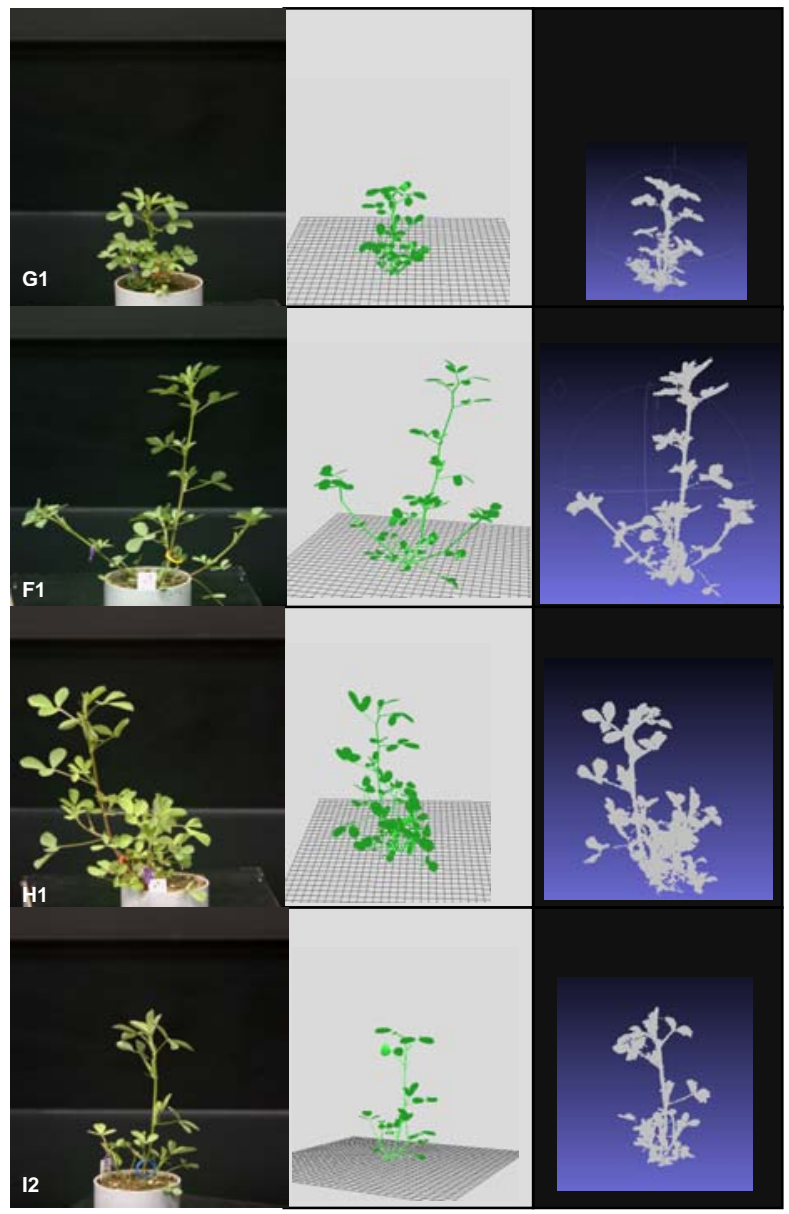

Figure 1: Pictures, corresponding 3D digitized plants and laser scanner point clouds for four contrasting alfalfa plants (LD, D1).

\subsection{Laser scanner point clouds acquisition}

For each of the digitised plants, 3D point clouds were acquired indoor using a rapid non-contact 3D laser scanner (VIVID 910 ), Konica Minolta Holdings Inc., Tokyo, Japan). Focal distance (TELE, $\mathrm{f}=25 \mathrm{~mm}$ ) and plant distance from the scanner $(2.25 \mathrm{~m})$ provided an about $1 \mathrm{~mm}$ resolution. Plants were placed on a synchronised rotary table, so that three views were captured modulo $120^{\circ}$. They were merged without outlier processing with the "Rapid Form" software (Inus Technology Inc., Seoul, South Korea).

\subsection{D point cloud processing}

Point clouds were further processed with the open source Meshlab software [13].

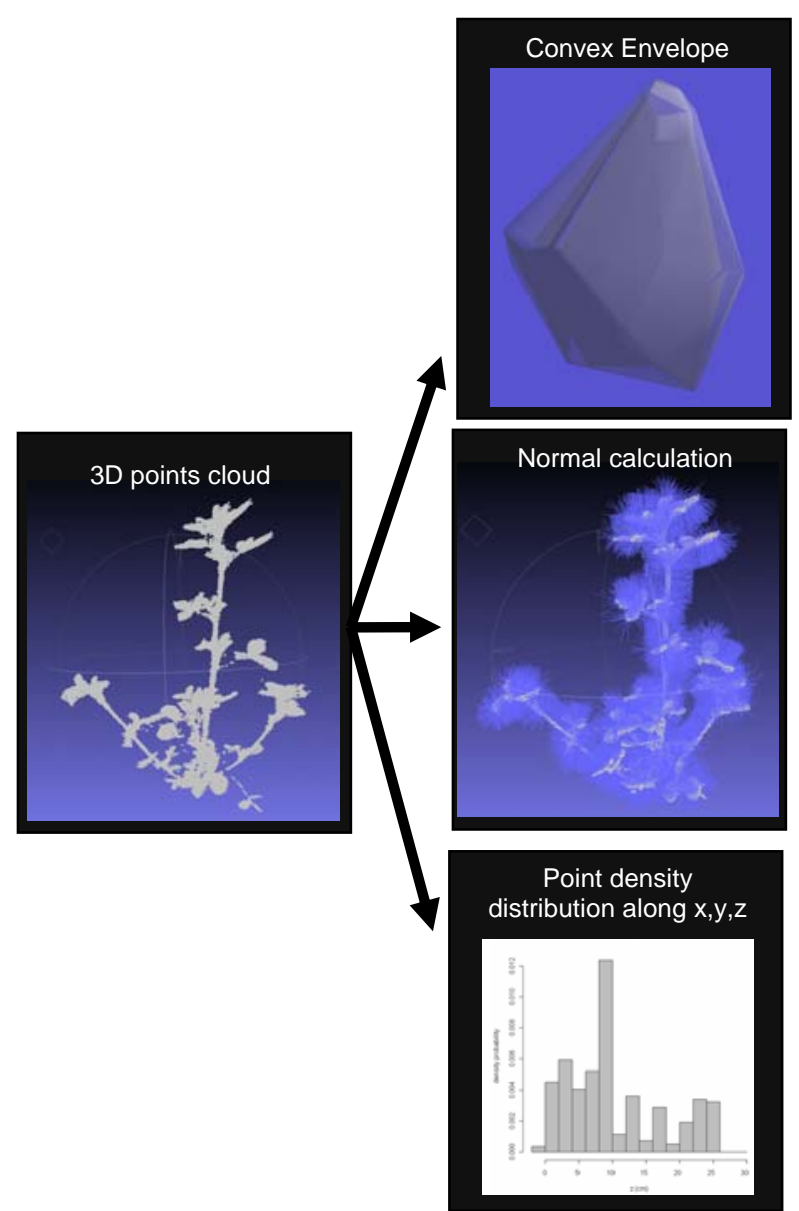

Figure 2: Illustration of the whole plant properties extracted from the 3D point clouds. 
Plant characteristics necessary to calculate light interception with a turbid medium approach were estimated (Fig. 2). First, point density distributions were established along $\mathrm{x}, \mathrm{y}$ and $\mathrm{z}$ axes. Second, an oriented point cloud was calculated using the algorithm computing vertices normals without exploiting mesh connectivity. The ten closest neighbour points were determined and used to fit a plane that gives an estimate of the normal of the point. The distribution of normal elevations was then calculated. Finally, the Ball Pivoting algorithm enabled to reconstruct a raw 3D mesh from the oriented point cloud [14]. Each plant convex boundary envelope was defined by fitting a convex hull to this mesh using PlantGL [11].

\subsection{Assessment of the derived leaf properties}

Total numbers of scanned points, their relative distributions in space as well as the distribution of normal elevations were compared to total leaf area, plant surface distributions and plant surface elevations extracted from the corresponding 3D digitizations. Ultimately, light interception properties of the turbid medium generated with these plant characteristics (i.e. boundary envelope, leaf area, 3D spatial distribution functions and leaf angles distribution) were compared to the light interception of the digitizations. A statistical envelope-based reconstruction method was used to build-up 3D plant turbid medium [8, 15]. Individual plant representations were gathered according to their position and orientation in the actual stand to build up virtual canopies.

\section{Results and Discussions}

Figure 3 presents the relationship between the number of scanned points for a plant and the measured plant leaf area. For a given scanner configuration, the two variables were strictly correlated, making it possible to infer total leaf area from the number of scanned points. The spatial distribution of scanned points also appeared as a very good proxy for the spatial distribution of leaf area. Relative vertical distributions were for instance similar for total plant area (leaves + stems) and for scanned points (Fig. 4). Considering leaves only (L) instead of leaves + stems (LS) surfaces did not modify the relative spatial distributions of plant surfaces (not shown). Discrepancies were frequently more important for leaf angle distributions between scanner data and digitisations (Fig. 4). In most cases, the distributions of elevation angles were quite similar between LS digitizations and scanned point's normals. They were intermediate between classical planophile and

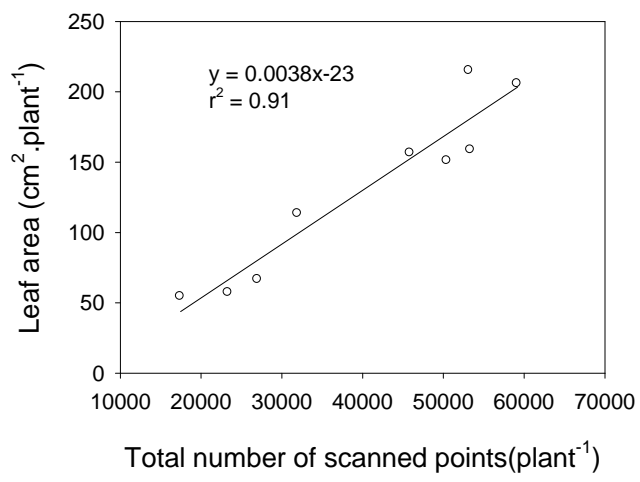

Figure 3: Relationship between total number of scanned points and plant leaf area (LD, D1).

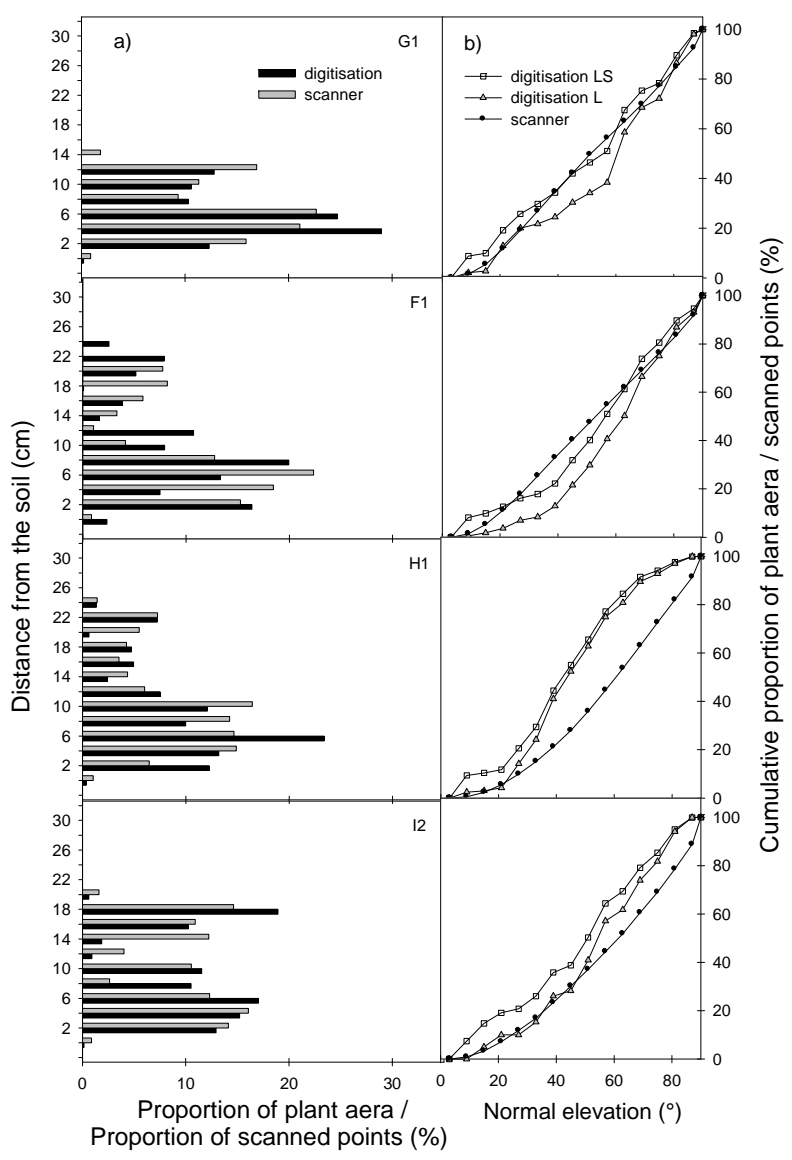

Figure 4: Comparisons of vertical distributions of scanned points and whole plant surfaces (a) and of cumulative leaf elevation distributions for scanner data and magnetic digitizations (b) for the four plants presented in Fig.1. 
plagiophile distributions [16]. It however also happened that the angle distributions clearly differed between the two types of characterisation. Although deeper flaws and/or inappropriate sampling of the scanner method can not be excluded, the most plausible reason for that could be the time delay between scanner acquisition and digitisation that could allow leaflet reorientation for the last digitised plants. The more plagiophile distributions observed for some digitizations indeed seem to correspond to plants adapted to indoor light conditions.

The overall consequence of these plant characterisations was finally assessed on the light interception properties of the plants (Fig. 5). Interestingly, the data extracted from the point clouds enabled the reconstruction of 3D plants that provided good estimates of light interception as compared to the digitizations. The accuracy required for such a calculation of light partitioning was thus met by using raw scanner point clouds. In a previous analysis, total plant leaf area and the relative vertical distribution of leaf area were shown to have the more influence on light partitioning among individuals within a canopy [8]. The method thus appears promising for nondestructive follow-up of plant architectures on a large scale. It could probably be applied to point clouds obtained from other devices (e.g. time of flight camera, structured light scanner...). The method is currently being tested on a broader range of plant architectures, including plants with a high degree of self-shading.

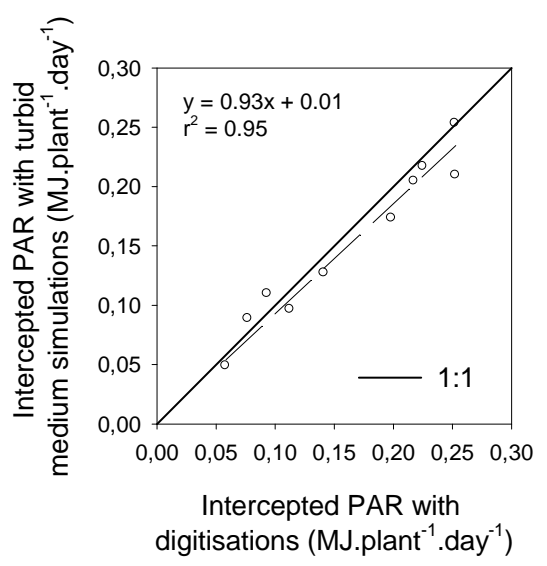

Figure 5: Comparison of daily light interceptions between 3D digitized plants and turbid-mediums reconstructed from scanner data (LD, D1).

\section{Acknowledgments}

This study was supported by the Conseil Régional Poitou-Charentes (http://www.poitou-charentes.fr) and by Agropolis Foundation (PlantScan3D Project). We would like to thank Dobrina Boltcheva for fruitful discussions.

\section{References}

[1] F. Tardieu, and R. Tuberosa, 2010. "Dissection and modelling of abiotic stress tolerance in plants." Current Opinion in Plant Biology 13(2): 206-212.

[2] Y. Chéné, D. Rousseau, et al. 2012. "On the use of depth camera for 3D phenotyping of entire plants." Computers and Electronics in Agriculture 82(0): 122-127.

[3] A. Paproki, J. Fripp, et al. 2011. "Automated 3D segmentation and analysis of cotton plants." In: Digital image computing techniques and applications(DICTA), 555560.

[4] C. Preuksakarn, F. Boudon, P. Ferraro, J-B. Durand, E. Nikinmaa, C. Godin, 2010. "Reconstructing plant architecture from 3D laser scanner data”. In: Proceedings of the 6th International Workshop on Functional-Structural Plant Models (FSPM), 14-16.

[5] S. Delagrange and P. Rochon, 2011. "Reconstruction and analysis of a deciduous sapling using digital photographs or terrestrial-LiDAR technology" Annals of Botany 108(6): 991-1000.

[6] R.B. Rusu and S. Cousins, 2011. "3D is here: Point cloud library (PCL). In: IEEE Int. Conf. on Robotics and Automation (ICRA) ", pages 1-4, Shanghai, China.

[7] R.A. Duursma, D.S. Falster et al. 2012. "Light interception efficiency explained by two simple variables: a test using a diversity of small- to medium-sized woody plants”. New Phytologist 193(2): 397-408.

[8] G. Louarn, D. Da Silva, C. Godin and D. Combes, 2012. "Simple envelope-based reconstruction methods can infer light partitioning among individual plants in sparse and dense herbaceous canopies”, Agricultural and Forest Meterology, In Press

[9] H. Sinoquet and P.Rivet, 1997. "Measurement and visualisation of the architecture of an adult tree based on a three-dimensional digitizing device”. Trees 11:265-270.

[10] R. Barillot, G. Louarn, A.J. Escobar-Gutiérrez, P. Huynh and D. Combes, 2011. "How good is the turbid medium-based approach for accounting for light partitioning in contrasted grass-legume intercropping systems?” Annals of Botany, 108(6) 1013-1024. 
[11] C. Pradal, F. Boudon, C. Nouguier, J. Chopard and C. Godin, 2009. "PlantGL: a Python-based geometric library for 3D plant modelling at different scales”. Graphical Models 71(1): 1-21.

[12] C. Pradal, S. Dufour-Kowalski, F. Boudon, C. Fournier and C. Godin, 2008. "OpenAlea: a visual programming and component-based software platform for plant modelling”. Functional Plant Biology 35(10): 751-760.

[13] P. Cignoni, M. Callieri, M. Corsini, , M. Dellepiane, F. Ganovelli and G. Ranzuglia, 2008. "Meshlab: an opensource mesh processing tool”. Euro. Ital. Ch. Conf. 129-136.

[14] F. Bernardini, J. Mittleman, H. Rushmeier, C. Silva and G. Taubin, 1999. "The ballpivoting algorithm for surface reconstruction” IEEE Transactions on Visualization and Computer Graphics, 5(4):349-359.

[15] G. Louarn, D. Combes, D. Da Silva and C. Godin, 2010. "Reconstruction methods to estimate light partitioning among individual plants in crowded canopies", In: Proceedings of 6th international workshop on Plant Structural-Functional Models, Davies, CA, pp 74-76.

[16] H. Sinoquet and B. Andrieu, 1993. "The geometrical structure of plant canopies: Characterization and direct measurements methods”. In: Varlet-Grancher, C., Bonhomme, R. and Sinoquet, H Editors, 1993. Crop structure and light microclimate: characterization and applications INRA, Paris, pp. 131-158. 